\title{
Global Incidence and Mortality Rates of Stomach Cancer and the Human Development Index: an Ecological Study
}

\author{
Salman Khazaei ${ }^{1}$, Shahab Rezaeian ${ }^{2}$, Mokhtar Soheylizad ${ }^{1}$, Somayeh Khazaei ${ }^{3}$, \\ Azam Biderafsh ${ }^{4 *}$
}

\begin{abstract}
Background: Stomach cancer (SC) is the second leading cause of cancer death with the rate of $10.4 \%$ in the world. The correlation between the incidence and mortality rates of SC and human development index (HDI) has not been globally determined. Therefore, this study aimed to determine the association between the incidence and mortality rates of SC and HDI in various regions. Materials and Methods: In this global ecological study, we used the data about the incidence and mortality rate of SC and HDI from the global cancer project and the United Nations Development Programme database, respectively. Results: In 2012, SCs were estimated to have affected a total of 951,594 individuals (crude rate: 13.5 per 100,000 individuals) with a male/female ratio of 1.97 , and caused 723,073 deaths worldwide (crude rate: 10.2 per 100,000 individuals). There was a positive correlation between the HDI and both incidence $(r=0.28, P<0.05)$ and mortality rates of $S C(r=0.13, P=0.1)$ in the world in 2012. Conclusions: The high incidence and mortality rates of SC in countries with high and very high HDI is remarkable which should be the top priority of interventions for global health policymakers. In addition, health programs should be provided to reduce the burden of this disease in the regions with high incidence and mortality rates of SC.
\end{abstract}

Keywords: Stomach cancer - human development index - incidence - ecological study

Asian Pac J Cancer Prev, 17 (4), 1701-1704

\section{Introduction}

Cancer is known as a major problem in human societies, so that accounts for the highest mortality after cardiovascular disease. In addition to the high mortality, associated with economic-psychological consequences and also lead to disabilities (Zhang et al., 2015; Di Pardo et al., 2016). Stomach cancer (SC) is the second leading cause of cancer deaths in the world; approximately $90 \%$ of SCs are adenocarcinoma with the high mortality rate. According to the statistics provided by the World Health Organization (WHO), $10.4 \%$ of the main causes of deaths due to SC and every year about 930000 people are diagnosed as new cases that at least 700,000 of them die because of this disease in the world (Parkin et al., 2005).

In worldwide, there is wide variation in the incidence and mortality rate of SC, and its prevalence in Japan, parts of South America and Eastern Europe is very high. In the United States, from 21500 new cases of SC, 13000 individuals led to death. As well as in countries such as China, Chile, Ireland, Costa Rica, North and South Korea, Finland and Iceland is prevalent and it seems that environmental differences over racial differences responsible for this significant difference (Brenner et al., 2009; Derakhshan et al., 2009). Studies have shown that the incidence and mortality of SC varies greatly from region to region (Jemal et al., 2010; Siegel et al., 2012), which can be indicative of the impact of lifestyle such as smoking, alcohol drinking, occupational and environmental exposures (Parkin et al., 2001). Other risk factors such as Helicobacter pylori (Uemura et al., 2001), blood group, age, sex, chronic gastritis have also been reported (Stewart and Kleihues, 2003; Jemal et al., 2010; Siegel et al., 2012; Wang et al., 2012). According to studies, many factors have impact on cancer. Human Development Index (HDI) is also one of the known risk factors as independent variable. Studies also have shown that the HDI is associated with the occurrence and distribution of various types of cancer (Kiadaliri, 2014; Guo et al., 2016; Khazaei et al., 2016). On the other hand, the correlation between the incidence and mortality rates of SC and HDI has not been globally determined.

Therefore, this study aimed to determine the association between the incidence and mortality rates of SC and HDI in various regions. To understand the role of the HDI in the incidence and mortality of SC around the world with

${ }^{1}$ Department of Educational Health, School of Public Health, Hamadan University of Medical Sciences, Hamadan, ${ }^{2}$ Social Development \& Health Promotion Research Center, Gonabad University of Medical Sciences, Gonabad, ${ }^{3}$ Operating Room, Rafsanjan University of Medical Sciences, Rafsanjan, ${ }^{4}$ Department of Social Medicine, Qom University of Medical Sciences, Qom, IR Iran *For correspondence: abiderafsh@yahoo.com 


\section{Salman Khazaei et al}

effective health planning by health policy and preventive control measures can effectively reduce the incidence of and deaths from this disease.

\section{Materials and Methods}

This is an ecological study on the relation of the agespecific incidence and mortality rate (ASR) of SC and HDI. HDI has several main components that include: life expectancy at birth, mean years of schooling, and gross national income (GNI) per capita; and also some ancillary indexes including: Percent of urbanization, and Age standardized obesity (defined as BMI $>30$ ) in adults.

Data about the ASR for 184 countries about incidence and mortality rate of SC for the year 2012 was obtained from the global cancer project available at http:// globocan. iarc.fr/Default.aspx. Data about the HDI and other indices were obtained from the United Nations Development Programme database.

Including to the analysis was restricted to countries for which both the epidemiological data from the GLOBOCAN database and HDI were available. In this study, we used the correlation bivariate method for assessment of the correlation between the incidence and mortality rates of SC and the HDI. We also used linear regression models for assessment of the HDI effect on SC occurrence rates. The two-sided level of statistical significance was considered less than 0.05. Data were analyzed by Stata computer software version 12 (StataCorp, College Station, TX, USA).

\section{Results}

In 2012, SCs were estimated to have affected a total of 951,594 individuals (crude rate: 13.5 per 100,000 individuals) with male/female ratio of 1.97 , and caused
723,073 deaths worldwide (crude rate: 10.2 per 100,000 individuals). SC was ranked as the 5 th of most common cancers among both sexes by GLOBOCAN database, by $6.8 \%$ of total cancers incident.

The highest HDI index values were belonged to privileged countries such as USA, Canada, Australia, and Western Europe (Figure 1). In addition, Figure 2 shows the estimated incidence of SC worldwide. Accordingly, $\mathrm{SC}$ incidence rate occurred most frequently in the East of

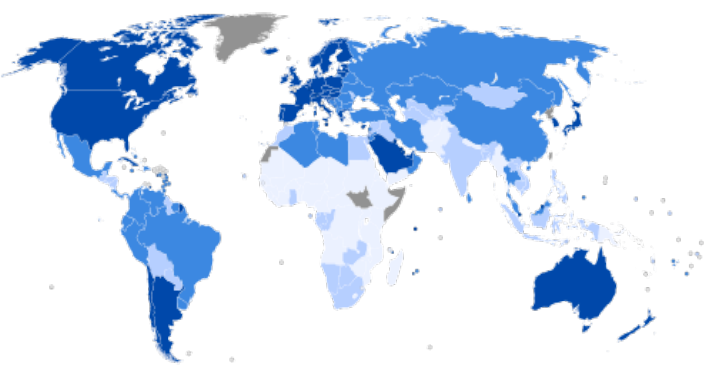

Figure 1. World Map Indicating the Categories of Human Development Index by Country (based on 2013 data)

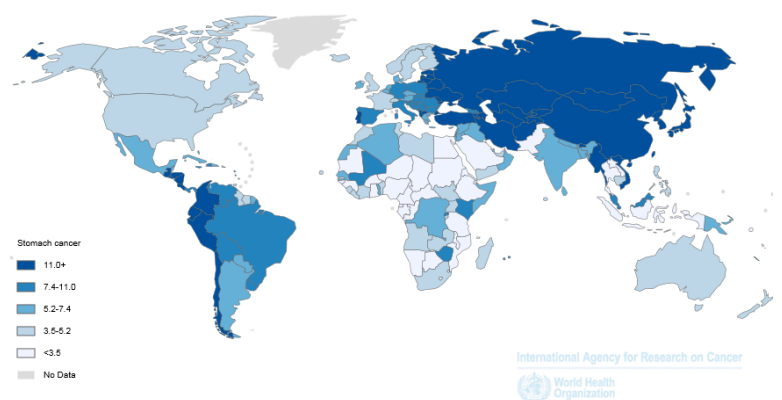

Figure 2. Incidence Age-Standardized Rates per 100,000 of Stomach cancer in the world in 2012

Table 1. Incidence and Mortality Rates of Stomach cancer and HDI Component in Different HDI regions in 2012

\begin{tabular}{|lcccccccc|}
\hline \multirow{2}{*}{\multicolumn{1}{c}{ Region }} & \multicolumn{2}{c}{$\begin{array}{c}\text { Stomach cancer } \\
\text { Incidence }\end{array}$} & \multicolumn{2}{c}{$\begin{array}{c}\text { Stomach cancer } \\
\text { Mortality }\end{array}$} & \multicolumn{3}{c|}{ HDI Component } \\
\cline { 2 - 9 } & CR & ASR & CR & ASR & LEB & MYS & GNI & HDI \\
\hline Very High Human Development & 10.4 & 5.4 & 6.6 & 3.17 & 80.2 & 11.7 & 40,046 & 0.89 \\
High Human Development & 17.5 & 9.38 & 11.6 & 5.9 & 74.5 & 8.1 & 13,231 & 0.74 \\
Medium Human Development & 8.9 & 9 & 7.6 & 7.74 & 67.9 & 5.5 & 5,960 & 0.61 \\
Low Human Development & 2.07 & 4 & 1.98 & 3.9 & 59.4 & 4.2 & 2,904 & 0.49 \\
P-value & $<0.001$ & $<0.001$ & $<0.001$ & $<0.001$ & $<0.001$ & $<0.001$ & $<0.001$ & - \\
\hline
\end{tabular}

CR: Crude Rate, ASR: Age-Standardized Rates per 100,000, HDI: Human Development Index, LEB: Life Expectancy at Birth, MYS: Mean years of schooling, GNI: Gross national income per capita

Table 2. Effect of HDI Components and Demographic Variables on Stomach Cancer Incidence and Mortality Rates

\begin{tabular}{|c|c|c|c|c|c|c|}
\hline \multirow{2}{*}{ Variable } & \multicolumn{3}{|c|}{ Stomach Cancer Incidence } & \multicolumn{3}{|c|}{ Stomach Cancer Mortality } \\
\hline & $\mathrm{B}$ & $\mathrm{CI}$ & P-value & B & CI & P-value \\
\hline Life Expectancy at Birth & 0.2 & $(0.1,0.3)$ & $<0.001$ & 0.08 & $(0.007,0.16)$ & 0.033 \\
\hline Mean years of schooling & 0.6 & $(0.31,0.9)$ & $<0.001$ & 0.24 & $(0.016,0.47)$ & 0.036 \\
\hline Gross national income per 1000 capita & 0.05 & $(-0.05,0.09)$ & 0.85 & 0.03 & $(-0.007,0.04)$ & 0.083 \\
\hline HDI & 10.9 & $(5.17,16.8)$ & $<0.001$ & 3.7 & $(-0.8,8.1)$ & 0.1 \\
\hline Urbanization level (\%) & 0.05 & $(0.01,0.09)$ & 0.012 & 0.01 & $(-0.2,0.04)$ & 0.53 \\
\hline Age standardized obesity in adults & 0.04 & $(-0.05,0.12)$ & 0.38 & 0.25 & $(-0.04,0.09)$ & 0.46 \\
\hline
\end{tabular}




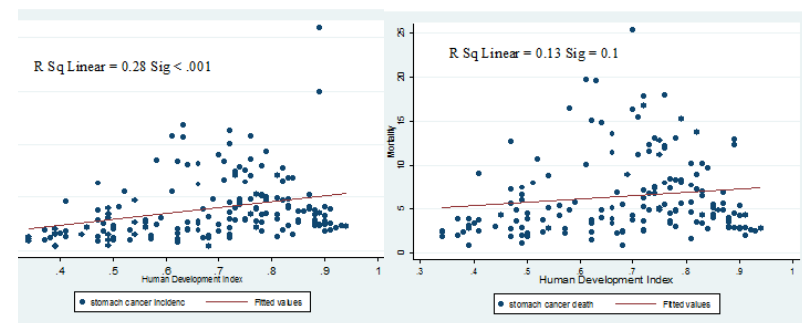

Figure 3. Correlation between the Human Development Index and stomach cancer incidence and mortality rates in the world in 2012

Europe and Asian countries.

While the highest age-standardized incidence of SC was seen in very high human development regions, also the highest age-standardized mortality of SC was belonged to these regions. More specifically, in high or very high developed regions, the highest value of LEB, MYS, GNI, and total HDI was estimated to be $80.2,11.7,40$, and 0.89 ; respectively (Table 1).

Figure 3 shows a positive correlation between the HDI and both incidence $(\mathrm{r}=0.28, \mathrm{P}<0.05)$ and mortality rates of SC $(r=0.13, P=0.1)$ in the world in 2012 .

Linear regression model showed that the mean of life expectancy at birth, mean years of schooling, HDI, and percent of urbanization have a positive effect on increasing in both incidence rate. Moreover, there was found a significant association between the mean of life expectancy at birth, mean years of schooling and mortality rate of SC (Table 2).

\section{Discussion}

The results showed that the cause of death in 723073 people was due to SC in the world in 2012. The crude mortality rate of SC was 10.2 per 100,000 which was consistent with results of a study conducted in 2008 (Da $\mathrm{Hu}$ et al., 2013). This study showed that SC ranks fifth among both sexes with $6.8 \%$ of all cancers. The highest HDI was belong to countries like the United States, Canada, Australia and Western Europe which are areas with very high HDI. The results showed that SC in the West Europe and Asian countries occurred frequently and the highest age-standardized incidence of SC has been reported in areas with high development indicators, which are consistent by the results of other studies that reported a high incidence of SC in developed countries than in developing ones (Youlden et al., 2008).

Both the age-standardized incidence and mortality rates were almost half of crude incidence and mortality rates, which these results show the effect of age on the incidence and mortality rates. There was a positive correlation between HDI and incidence of SC $(r=0.28)$ as well as the mortality rate of SC $(r=0.13)$ worldwide, in 2012. That is consistent with the results of other studies that have shown a positive correlation between HDI and cancer (Bray et al., 2012; Pakzad et al., 2015). The highest incidence rate reported in high developed regions than in other regions. Age-standardized mortality rate in regions with very high HDI is lower than in other regions, in the event that crude mortality rate in regions with low HDI, the lowest value is reported. These results can be derived from two factors, effective healthcare system resulting in earlier diagnosis and lower mortality in countries with very high HDI, and lower survival rate in countries with medium and low HDI or due to different effect of age on mortality in countries with very high and low HDI. The results are inconsistent with the results of a study conducted in 2013, linear and nonlinear regression analysis in the study showed an inverse relationship between SC and HDI, thus in high developed regions MIR (mortality to incidence ratio) have been reported low. This inverse relationship also shows that existence of effective health care system in high developed regions resulted in the low MIR ( $\mathrm{Da}$ Hu et al., 2013).

In a study conducted in 2013 reported that 28.8 million people have been diagnosed over 5 years as a cancer patient worldwide, nearly half of them live in regions with very high HDI which is only one-sixth of the world's population. In contrast, 3.4 billion people lived in countries with medium and low HDI while only had slightly more than a third of the world's cancer. Therefore, this difference may be due to effective healthcare system (Da Hu et al., 2013).

The results also showed that there was a significant difference between the incidence and mortality rates in regions with very high and high HDI. In this way, high developed regions have less incidence and mortality rates than developed regions which can be due to an increase in the incidence of other cancers. The results of a study conducted by Bray et al. based on longitudinal data from cancer registries with high quality is pretty consistent which showed that in regions with medium and high HDI, there was a reduction in the incidence of SC than regions with low HDI (Bray et al., 2012).

Linear regression model showed that the various components of the HDI have a positive effect on the increased incidence and mortality of SC. Life expectancy at birth increased by 1 unit, on average, increases incidence rates up to 0.2 and mortality rate up to 0.08 and in high developed countries to less developed regions, the role of more life expectancy in increasing the incidence and mortality rated has been reported; which could be due to people's survival as a result of higher life expectancy and therefore more likely to develop cancer (Yancik, 2005). HDI increased by 1 unit, on average, increases incidence and mortality rates up to 10.9 and 3.7, respectively. The mean years of schooling has strongest significant effect on the incidence and mortality risk at 0.6 and 0.24 , respectively. With increasing level of health literacy and awareness of early symptoms of SC and early detection, the incidence will increase. The impact of increasing agestandardized obesity on SC incidence and mortality risk was not significant. The incidence and mortality rates of $\mathrm{SC}$ was found to increase with increasing the percentage of urbanization. With increasing the level of urbanization, exposure to risk factors for SC will increase. Studies have shown that cancer is common in high income countries (La Vecchia, 2016). An increase of 1 unit per GDI increasing mean of incidence by 0.05 non-significantly and increasing mean of mortality by 0.03 significantly. This component 


\section{Salman Khazaei et al}

can also be associated with people afford to pay to participate in screening programs and access to these facilities which leading to an increase in the incidence and mortality rates.

One limitation of this study was impossible to generalize the results to small communities. It is recommended that similar studies be done at the national level and smaller communities in different countries. It also recommended that in future studies, other aspects related to $\mathrm{SC}$ to be surveyed.

In conclusion, According to the study, the high incidence and mortality rates of SC in countries with high and very high HDI is remarkable which should be the top priority of interventions for global health policymakers. In addition, the health programs should be provided to reduce the burden of this disease in the regions with high incidence and mortality rates of SC.

\section{Acknowledgements}

The data of the GLOBOCAN and the United Nations Development Programme (UNDP) were used for this study. This research has not received any specific fund. The authors declare that they have no conflicts of interest.

\section{References}

Bray F, Jemal A, Grey N, et al (2012). Global cancer transitions according to the Human Development Index (2008-2030): a population-based study. Lancet Oncol, 13, 790-801.

Brenner H, Rothenbacher D, Arndt V (2009). Epidemiology of stomach cancer. Methods Mol Biol, 472, 467-77.

Da Hu Q, Zhang Q, Chen W, et al (2013). Human development index is associated with mortality-to incidence ratios of gastrointestinal cancers. World J Gastroenterol, 19, 5261-70.

Derakhshan MH, Liptrot S, Paul J, et al (2009). Oesophageal and gastric intestinal-type adenocarcinomas show the same male predominance due to a 17 year delayed development in females. Gut, 58, 16-23.

Di Pardo BJ, Bronson NW, Diggs BS, et al (2016). The Global Burden of Esophageal Cancer: A Disability-Adjusted LifeYear Approach. World J Surg, 40, 395-401.

Guo Y, Zeng H, Zheng R, et al (2016). The association between lung cancer incidence and ambient air pollution in China: A spatiotemporal analysis. Environ Res, 144, 60-5.

Jemal A, Center MM, DeSantis C, et al (2010). Global patterns of cancer incidence and mortality rates and trends. Cancer Epidemiol Biomarkers Prev, 19, 1893-907.

Khazaei S, Rezaeian S, Khazaei Z, et al (2016). National breast cancer mortality and incidence rates according to the human development index: an ecological Study. Adv Breast Cancer Res, 5, 30-6.

Kiadaliri AA (2014). Gender and social disparities in esophagus cancer incidence in Iran, 2003-2009: a time trend provincelevel study. Asian Pac J Cancer Prev, 15, 623-7.

La Vecchia C (2016). Ovarian cancer: epidemiology and risk factors. Eur J Cancer Prev, [epub ahead of print].

Pakzad R, Mohammadian-Hafshejani A, Ghoncheh M, et al (2015). The incidence and mortality of lung cancer and their relationship to development in Asia. Transl Lung Cancer Res, 4, 763-74.

Parkin DM, Bray F, Ferlay J, et al (2005). Global cancer statistics, 2002. CA Cancer J Clin, 55, 74-108.

Parkin DM, Bray FI, Devesa SS (2001). Cancer burden in the year 2000: the global picture. Eur J Cancer, 37, 4-66.

Siegel R, Naishadham D, Jemal A (2012). Cancer statistics, 2012. CA Cancer J Clin, 62, 10-29.

Uemura N, Okamoto S, Yamamoto S, et al (2001). Helicobacter pylori infection and the development of gastric cancer. $N$ Engl J Med, 345, 784-9.

Wang Z, Liu L, Ji J, et al (2012). ABO blood group system and gastric cancer: a case-control study and meta-analysis. Int J Mol Sci, 13, 13308-21.

Yancik R (2005). Population aging and cancer: a cross-national concern. Cancer J, 11, 437-41.

Youlden DR, Cramb SM, Baade PD (2008). The international epidemiology of lung cancer: geographical distribution and secular trends. J Thorac Oncol, 3, 819-31.

Zhang X, Yan Y, Li S, et al (2015). Cancer burden in China from 2006 to 2010. Int J Clin Exp Pathol, 8, 13323-30. 